\title{
SEMIÓTICA DE LA ACTUALIDAD Y SEMIÓTICAS ACTUALES
}

\author{
SEMIOTIC OF CURRENT AFFAIRS AND CURRENT SEMIOTICS
}

\author{
Francisco LINARES ALÉS \\ Universidad de Granada \\ flinares@ugr.es
}

\begin{abstract}
Resumen: Se explora en este trabajo las posibilidades de que la disciplina semiótica elabore el concepto actualidad explicando e integrando en su corpus teórico y analítico el uso corriente del término. Depende en buena medida del concepto lingüístico y semiótico de actualización discursiva, y en razón de él es concebible hablar con rigor de semióticas actuales, o sea, discursos actuales. Al constituirse estas de manera jerárquica y cambiante en sus momentos de vigencia, se ve preciso que la Semiótica, en diálogo con otras disciplinas, diseccione críticamente el devenir histórico de sus actualidades. Así, desde un plano teórico analítico más general, se propone una explicación del carácter de los discursos contemporáneos y se ejemplifica en la forma en que en ellos queda involucrado el cuerpo humano. Con las conclusiones se pone de relieve que esta es una vía, entre otras posibles, por la que la Semiótica alcanza actualidad.
\end{abstract}

Palabras clave: Semiótica. Actualidad. Sociocrítica. Cuerpo. Crisis de lo simbólico.

\begin{abstract}
This paper explores the possibilities for the Semiotic to elaborate the concept of current affairs by explaining and integrating the common use of the term into its theoretical and analytical corpus. It depends to a large extent on the linguistic and semiotic concept of actualization, and because of this it is conceivable to speak rigorously of current semiotics, that is, current discourse. As these are constituted in a hierarchical and changing manner in their moments of validity, it is necessary for Semiotics, in dialogue with other disciplines, to critically dissect the historical development of their current discourse. Thus, from a more general analytical theoretical level, an explanation of the character of contemporary discourses is proposed and the way in which the human body is involved in them is exemplified. The conclusions highlight the fact that this is one way, among others, by which Semiotics can achieve actuality.
\end{abstract}

Keywords: Semiotics. Actuality. Sociocriticism. Body. Crisis of the symbolic.

\section{INTRODUCCIÓN}

El presente trabajo lleva por título "Semiótica de la actualidad y semióticas actuales" porque responde al propósito de reflexionar sobre la actualidad y lo actual en clave 
semiótica. Se ha de entender por semiótica tanto la actividad sígnica o producción discursiva del ser humano, como la disciplina llamada Semiótica. Esta es una semiótica especializada en asumir explicativamente cualquier semiótica. Por dicha asunción, la Semiótica es metasemiótica, del mismo modo que la Lingüística es metalenguaje.

Viene al caso porque se ve necesario trabajar sobre la hipótesis de que la semiótica (las semióticas y luego la disciplina que las estudia) no solo ha señalado lo actual, sino que ha forjado históricamente una idea de lo actual y de la actualidad. Es decir, la hipótesis de que no solo ha llevado a cabo una praxis demarcativa de actualidad - actual y actualidad son vocablos de significado crono-situacional, pues se refieren a lo que existe, o sucede, en el tiempo en que se habla y se profieren tales palabras - sino una conciencia o significación históricamente cambiante de lo actual. Dando un paso más, y ya adentrándose bajo la luz de la disciplina Semiótica, cabe contemplar también el hecho y la explicación de que a las semióticas o discursos se les atribuya la condición de actuales (o inactuales, claro está). Por último, la misma Semiótica ha de reflexionar internamente sobre su carácter actual o su actualidad. Entre otras cuestiones, puede plantearse que su actualidad dependa de un abordaje de los que percibe como discursos actuales. Todo ello sin adentrarse en el extenso campo de la semiótica del tiempo, donde habría que comenzar por la representación del tiempo mediante el lenguaje.

El reparo más inmediato que pueden recibir tales planteamientos es el de que lo evidente no necesita de mayores análisis, y que todo acaecimiento es actual en el momento en que capta nuestra atención o lo convertimos en objeto de interés. Anticiparse a dicho reparo equivale a dejar sentado que es precisamente esta evidencia la que hay que explicar semióticamente recorriendo el camino inverso: habría que ver qué condiciones -físicas, sociales, discursivas - se tienen que dar para que se perciba la entidad y los márgenes de lo actual. Y estas no se pueden dar sin una conciencia y una memoria de la historia individual y colectiva que posibilita dicha percepción. El comienzo es la misma idea de actualidad. El proceso parece — solo parece - sencillo: el signo actualidad convenido hasta cierto punto y cambiante, sirve para señalar fases del desenvolvimiento de las cosas desde la perspectiva también cambiante de quien o quienes las perciben. El significado situacional que dijimos que posee, hace de él un signo muy lábil, pero al mismo tiempo representativo de variadas posiciones físicas, sociales y discursivas. Ocurre algo semejante con términos como yo o nosotros, confrontables con el otro o los otros, como lo actual con lo inactual.

Teórica y metodológicamente, dado el alcance general del planteamiento, se tendrán en cuenta diversas aportaciones semióticas, desde la teoría de los procesos semiósicos de Peirce, a concepciones greimasianas y lotmanianas, así como la Sociocrítica, que integra también la perspectiva culturológica e historiográfica. La metodología investigadora que se sigue parte del estudio léxico y de historia de las ideas; en un segundo momento se centra en la explicación y desarrollo de conceptos semióticos atingentes a la actualidad; en una tercera fase, tomando la teoría sociocrítica sobre diversos regímenes históricos del 
signo, se reflexiona sobre las formas de representación actuales, ejemplificadas en una cuestión de actualidad como es la del cuerpo humano.

\section{LA ACTUALIDAD SEGÚN EL DISCURSO Y LA SEMIÓTICA}

\subsection{Formación de las nociones de actual y actualidad}

La actualidad no es meramente lo que acontece o acaba de darse en el momento en que se habla, como leemos en el DLE - por ejemplo, lo que ocurre o se dice en estos momentos y a lo que por tanto me pudiera referir ahora, o lo que ocurrió en otro tiempo que alguien refirió llamándolo su actualidad-, sino que actualidad es una noción, que forma parte de un paradigma, que tiene una vertiente categorial, un carácter evaluativo, e incluso se podría plantear como ideologema (Cros, 2009: 211-216). Con todo esto hay base para que sea abordable semióticamente, y conviene comenzar por revisar la historia de la noción, que ha de hacerse dentro de la historia cultural occidental donde se ha gestado. Profundizar en la averiguación de la conciencia y la vivencia, por tanto, de lo que los romanos llamaban praesens; de lo actual-is para los medievales (actual se atestigua en español en 1460) frente a la idea de inmovilidad celestial, etc.; porque cada mentalidad tiene su forma de establecer hitos en la continuidad de las cosas, de integrar lo extraordinario en lo ordinario. Para dicha profundización es necesario interrogar a los discursos, comenzando por el uso que hacen del término actualidad.

El sustantivo actualidad no se recoge aún en el Diccionario de Autoridades, publicado entre 1726 y 1739 , y en el primer Diccionario de la $R A E$ de 1780 ya se define, aunque parcialmente. En la mayoría de los casos recogidos en el CORDE del periodo comprendido entre 1783 y 1850, el sintagma preposicional de actualidad suele significar 'actual' y es, por tanto, equivalente a ese adjetivo. Sin embargo, en dos casos aparece ya claramente con la función propia del sustantivo, y no es el término filosófico, sino que significa 'los hechos o cosas del presente'. En Estébanez Calderón se destacan las trivialidades de la actualidad, dándole una valoración negativa, pero en los casos de Mesonero Romanos y Joaquín Francisco Pacheco, con las construcciones interés de actualidad y fuerza de actualidad la idea de actualidad determina un tipo de interés y de fuerza. De este modo el sintagma de actualidad ya no significa meramente 'actual', sino 'de relevancia para la actualidad', como se ve en símil de actualidad.

La aparición de esta segunda acepción del sustantivo, tal como hemos visto, tiene seguramente que ver con el efecto de la prensa periódica en el siglo XIX, que relaciona claramente el objeto de la información con el interés, y más aún, con el interés común. Así define el DLE dicha segunda acepción: 'Cosa o suceso que atrae y ocupa la atención del común de las gentes en un momento dado'. Por tanto, no solo la palabra está ausente en el Diccionario de Autoridades, sino que la acepción más común de la palabra no aparece hasta avanzado el siglo XIX, en el contexto de una incipiente sociedad de la comunicación. 


\subsection{Conceptos semióticos}

Se ha visto en el punto anterior que es relevante epistemológicamente apostar por el principio de que la conciencia de la actualidad se ha venido formando según fases históricas. A partir de esa conciencia histórica de actualidad discursiva la Semiótica elabora - esta era nuestra segunda hipótesis - el concepto de actualidad. La Semiótica no solo la objetiva como hecho del discurso y contenido mental, sino que la adopta con cierto propósito. Es decir, la integra en su dispositivo conceptual y metodológico. Conviene precisar que es muy aventurado hablar de la disciplina Semiótica en general y de que integra sin más la noción de actualidad en su dispositivo conceptual. Lo cierto es que al menos la Semiótica contemporánea tanto de orientación peirceana como saussureana lo hace posible. Y esto es así, además, porque en la lógica de ambos está ya la cuestión de la posibilidad/suceso, o virtualidad/actualidad. La teoría y análisis del discurso hace por tanto posible objetivar y conceptualizar la actualidad discursiva. El dispositivo conceptual que lo hace posible pivota sobre las dicotomías sistema/proceso y cultura/extracultura en la forma en que los plantea I. Lotman. De este modo, no solamente puede explicar la génesis discursiva de la idea de actualidad, y formar un concepto de actualidad, sino que está en condiciones de teorizar y desvelar los mecanismos de lo que podemos llamar las semióticas o los discursos actuales (de actualidad en una etapa histórica determinada).

Se puede delimitar, pues, dentro del marco disciplinar de la Semiótica el concepto de actualidad (y actual) y el concepto de semiótica (s) actual (es). Esto teniendo en cuenta que esos dos conceptos solo balizan convencionalmente dentro de una gama de hechos o ideas. El primero (actualidad entendible dentro del sistema virtualidad/actualidad) opera como paso inicial y el segundo (entendible dentro del paradigma estructuración/reestructuración de los sistemas) permite explicar la actualidad de los discursos, del conjunto de discursos de una cultura. Uno es una herramienta para entender la historia, y el otro para entender la Historia con mayúscula. Laten aquí conceptos saussureanos (virtualidad/actualidad y sincronía/diacronía) pero diacronía tiene aquí ya el sentido de historia que básicamente es sucesión de estados de cosas.

\subsubsection{Actualización, actualidad}

En Semiótica y Lingüística, actualidad posee un significado más restringido de carácter técnico, que tiene que ver con el verbo actualizar y el sustantivo actualización, pero que sigue remitiendo a la idea de acto.

Partiendo de la dicotomía saussureana de potencia y acto en el lenguaje, para Greimas y Courtés (1982: 29) la actualización es el paso del sistema virtual al proceso real en el que se dan los signos. Esto, en un determinado sentido es el hacerse presente una "unidad de lengua" en un "contexto lingüístico" dado; es decir, la realización de la alternativa in 
absentia/in praesentia, paradigma/sintagma (1982: 29). Todo acto discursivo no solo es actuación sino actualización.

El concepto presenta una formulación particular para la semiótica de la narración. Greimas y Courtés a este respecto de los hechos del relato, utilizan una articulación ternaria de conceptos, y diferencian lo virtual/lo actual/lo realizado. Se podría reducir a dos dualidades: virtual/actual (lo juncionado); y actual no realizado (disjunción)/actual realizado (conjunción).

Si todo sujeto semiótico para su existencia necesita de la atribución de algún objetovalor, el discurso, cuando se realiza, suscita, entre otros, el objeto-valor actualidad, que se le puede reconocer bajo determinadas condiciones. Debemos tener en cuenta, además, que por el mecanismo de embrague/desembrague la temporalidad del discurso actualiza otras temporalidades en la medida que actualiza otros discursos. Esos otros discursos del pasado quedan así actualizados integrándose igualmente en el presente bajo los valores de interés y actualidad.

\subsubsection{Semióticas actuales}

Los actos - en realidad, en razón de lo dicho anteriormente, sistemas de actos discursivos o sistemas de hechos tematizados - poseen una incidencia que es espacial, temporal y de jerarquía; tanto para la mente individual como para la mente colectiva, y sus respectivas memorias. Y además unos niveles del discurso tienen más incidencia que otros. En síntesis, tematice o no la actualidad, valorice o no la misma, en la cultura siempre hay discursos que ocupan la posición de la actualidad, que es lo mismo que dejar a los demás en la inactualidad. Las llamaremos, desde el punto de vista del ahora, discursos o semióticas actuales. Y solamente a partir de ellos podemos procesar los que tuvieron cierta vigencia en otras fases de la cultura, convirtiendo desde nuestro ahora la inactualidad en actualidad.

En el contexto social-cultural cobra entonces pleno sentido la idea anterior de unión del sujeto discurso con el objeto valor actualidad. Es más, en esto hay una cierta performatividad, porque los discursos tienden a imponerse autoproclamándose actuales. Se da en complicidad con los destinatarios y con el soporte institucional sea de mentideros, púlpitos, medios de comunicación o redes sociales.

Para profundizar en esta teoría sobre los discursos actuales se ha de analizar críticamente no solo el desenvolvimiento de unos discursos con respecto a otros, sino cómo construyen la actualidad en diversos ámbitos. Por ejemplo, lo que los medios de comunicación masiva llaman actualidad (agenda setting) responde a la potestad que les es atribuida de representar a la misma (Wolf, 1987: 163-200). Pero la Semiótica debería intentar proponer cuál es el marco de actualidad en el que se dan todos ellos.

Así, otras preguntas quedan abiertas: ¿Qué marca el principio y el final del ahora en que determinados discursos están vigentes? Y después, ¿cómo explicar el cambio de unas vigencias a otras? 
Dentro de la gradual abstracción que va desde atender un discurso concreto - el editorial de un periódico esta mañana, por ejemplo - hasta la mayor abstracción del concepto de signo o de discurso, es posible detenerse en dos escalones, próximos a ambos extremos, en los que es perceptible la transformación histórica. Así, las opciones son las siguientes:

a) tomar en consideración a pequeña escala la pujanza de los discursos, de unos con respecto a otros, y atender a sus temáticas y tratamientos como fenómenos de actualidad (es conocida la estrategia de dar a conocer convenientemente asuntos para ocultar otros);

b) de manera más sistemática y abstracta, partiendo con mayor necesidad de los conceptos operativos de signo, sistema, discurso, estructura discursiva ... intentar detectar cuándo cambia el régimen del signo, los patrones discursivos de la significación, es decir, las formas de significar. La Semiótica tiene la ocupación de prestar atención a los discursos que tratan de imponerse en cada tiempo y lugar, pero también tiene que ocuparse de retroceder epistemológicamente a sus bases y pensar sobre las formas de significar y los cambios, como formas de cultura, en una perspectiva de largo alcance o gran periodización.

Evidentemente, en los dos casos se supone una perspectiva histórica — no podría ser de otro modo, entiéndase como se entienda la historia, aunque sea como mera diacroníaque además ha de ser social, pero en el segundo de los casos, el indicado como b), la Semiótica determina la actualidad que más específicamente le interesa: la aparición de cambios históricos en la operación misma del significar. Para esto se debe tener claro cuándo se detectan cambios sustanciales en el régimen del signo, aunque no haya un hiato perceptible y los cambios se produzcan durante una larga temporalidad.

En lo que sigue sobre el diagnóstico de actualidad más abstracto y de largo alcance, que será tratado en el apartado tercero, me basaré en Edmond Cros, quien plantea lo que entiende como formas históricas de representación, más concretamente matrices históricas de representación, y se pregunta sobre cuáles son las formas actuales de las mismas (las que parecen decantarse en la actualidad) (Cros, 2021).

\section{PROPUESTA SOBRE EL RÉGIMEN DEL SIGNO Y DE LOS DISCURSOS EN LA ACTUALIDAD}

Edmond Cros diferencia cuatro formas de representación dadas históricamente. El supuesto es que "cualquier representación en el mismo momento en que se da, actualiza una estructura discursiva virtual que estaba en espera de esta actualización" (2021: 18). Dichas estructuras discursivas virtuales se atienen a dispositivos matriciales que marcarían el conjunto del campo discursivo de toda una época de la historia de la cultura. 
Estas supondrían una serie de "rupturas históricas desde el siglo XVI hasta hoy día", a saber:

1. Una concepción sagrada del lenguaje; 2 . El descubrimiento del Otro y el anuncio de la arbitrariedad del signo; 3. El surgimiento de un nuevo lenguaje que cuestiona el significado; 4. La dialéctica de lo real y de lo virtual, y la disyunción que separa la realidad de su representación por el lenguaje (2021: 19).

Por supuesto, sus efectos se darían en todos los discursos, pero se puede ver ya en el lenguaje mismo como "sistema primero de representaciones de la realidad" (2021: 19). Nos interesan las fases tercera y cuarta, aunque el proceso que va de la primera a la segunda, descrita por Foucault en Las palabras y las cosas, es el que inspira la reflexión de Cros. Y sobre todo nos va a interesar la última de las dos, porque la matriz cultural más reciente sería la que explica las formas del discurso actual.

Pero antes, la episteme o matriz cultural que aparece desde mediados del siglo XIX, que se hace notar, por este orden, en los avances de la óptica, el psicoanálisis y la teoría de Saussure, se caracteriza por un cuestionamiento del significado referencial, en cuanto el significado no se da sino de manera inherente a las propias leyes del signo (Cros, 2021:17).

En esta episteme es donde se sitúan el estructuralismo y la semiótica (fundamentalmente saussureana, aunque Peirce habla también de la convencionalidad simbólica). Por tanto, la crisis de esta episteme tiene mucho que ver con la crisis del estructuralismo y la semiótica basada en dicha episteme. Así, en cierta Semiótica se rechaza la legalidad del signo por idealista y se defiende la adopción del significante, no en función de significados preestablecidos, sino que se explora su uso como representación inmediata de quien lo adopta. Se postula una superación de la diferencia, del desfase entre significante y significado que siempre supone una imposición. La liberación del signo de esas ataduras abre la posibilidad de forjar una nueva realidad. Así en la crítica de la economía del signo formulada por Ferruccio Rossi-Landi (1968); y sobre todo en el grupo Tel Quel, Barthes y Kristeva, quienes siguieron un camino de abierta crítica al postulado sígnico del que partían, apurándolo (Selden, 1987: 89-126; Ablali y Ducard, 2009: 109-119). La resistencia de Greimas y de Cesare Segre, entre otros que también defienden la entidad del texto y los límites de la interpretación, revelan la tesitura en que se desenvuelve la disciplina.

Dice Edmond Cros que en esta episteme "se agazapa la episteme que la va a desestructurar, como el capitalismo financiero se agazapa en las condiciones estructurales del capitalismo industrial" (Cros, 2021: 17) pues "se trata de algo virtual, pero no ha sido todavía asumido como tal". De modo que, coincidiendo con la pérdida del patrón oro en el intercambio monetario, "a partir de los años 70-80 se producirá la crisis que afecta al orden simbólico en numerosos puntos que han sido puestos de manifiesto por estudios concordantes sobre los medios". 
La práctica artística experimental, que ya había servido de estímulo para las concepciones formalistas y semióticas formuladoras de la convencionalidad del signo, propicia su crítica precisamente al profundizar en la idea del signo artístico como experiencia única, fundadora de realidad. Pero, en cualquier caso, si se exalta en extremo la presencia para convocar lo ausente - la otra cara, por definición, del signo-, se está desligando lo ausente de cierta legalidad que permite reconocerlo como significado y realidad, y se está descubriendo un flanco en el orden simbólico que va a pasar a ser ocupado por nuevos discursos. Con esta inestabilidad del signo, situacionalidad, volatilidad del significado, se va a señalar el lugar que tenderá a ocupar un nuevo simbolismo.

En la figura siguiente, mediante la ubicación de la representación diferenciada entre significante/significado dentro del cuadrado semiótico, se señala la contradicción de la tercera matriz. La negación de la diferenciación, expresada mediante la flecha que va de diferenciada a no diferenciada, permite por implicación la representación idéntica, característica de la matriz más reciente, la de la era virtual.

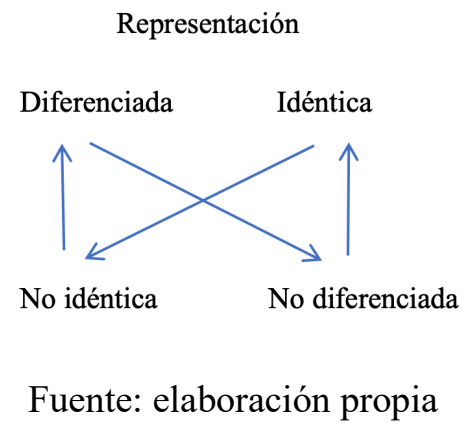

Describir en pocas frases la situación a la que parece que nos está trayendo esta crisis que se inició en los años setenta y ochenta, lo que Cros llama nueva episteme, es tarea imposible. Todo lo más se pueden dar algunas claves. Esta episteme que rige los discursos actuales es inseparable de la irrupción de la informática y la digitalización en el plano tecnológico, la mundialización de las relaciones y de la comunicación, que se corresponde en el plano económico por el predomino creciente del capitalismo financiero, una vez que se eliminó el patrón oro. Lo que esto reporta a la cultura es una autonomización de los sistemas, pues lo mismo que la economía tiene sus propias reglas y se tiene el convencimiento de que no cuenta su significación por relación a otros sistemas y valores de la cultura, se entiende que cualesquiera reglas son autosuficientes. Consiguientemente se degrada el carácter representativo del signo en general y la palabra.

El significado y la realidad ya no es representada por los signos como una alteridad con la que hay que contar, alteridad que es la razón de ser del signo, sino que el signo y su realización tiende a ser lo mismo ( $c f$. lo dicho en apartado 2 sobre potencialidad, actualidad y realidad). El signo no es una virtualidad, sino que se da como realidad. Realidad virtual es el ideologema que representa esta nueva situación de sustracción del 
significado. Se opera una regresión al iconismo, puesto que el icono produce la sensación de estar ante la realidad, lo que en términos peirceanos supone la dejación de lo simbólico y de la argumentación. Lo que Barthes proponía como el placer y el goce del signo por la necesidad de dotar al signo de una significación desalienante, va camino de convertirse en una alienante ludopatía social. El valor de uso esgrimido ha sido de nuevo sometido a la ley del mercado.

\section{UN ENCLAVE DE LAS SEMIÓTICAS ACTUALES: CUERPO, SUJETO Y SUJECIÓN}

Se propone en este apartado entrar en el discurso actual de manera algo más pormenorizada. No atendiendo a una modalidad o tipo de discurso - aunque también atañe a ello - sino a cómo el cuerpo afecta y se ve afectado por el discurso en esta forma de significar o episteme de la que antes se ha hablado, con el añadido de la coyuntura de la Covid-19.

Tenemos asumido que el cuerpo es el soporte biológico de la semiótica y de la cultura, que se convierte en verdadero productor y producto cultural desde el momento en que el sujeto se significa con él. El sujeto cultural da forma y sentido al cuerpo, como si fuera su texto (el sujeto es al cuerpo lo que la idea inspiradora o dianoia es al texto), y esto lo podemos decir sin necesidad de recurrir a la idea de un alma prevalente y trascendente. Pero el cuerpo también crea al sujeto cultural. Precisamente lo que propone el grupo Tel Quel, Barthes o Kristeva, es la idea de que algo cultural como el signo o el texto, sea la proyección simbólica del cuerpo, es decir, que el texto trabaje lo simbólico en función de las necesidades del cuerpo.

Mas debido a esa inversión del paradigma de la que se ha tratado en el apartado anterior, el cuerpo como significante tiende a identificarse con el significado y la realidad, por más que, como se ha dicho, el cuerpo sea una alteridad en la que el sujeto se reconoce, pero desde la que también se distancia culturalmente. Así, esa omnipresencia del significante cuerpo rompe la dualidad simbólica, y lo deja como signo y a la vez realidad. El sujeto que vive su cuerpo como signo-realidad experimenta una cierta enajenación, porque no distingue la representación, sobre todo la imagen icónica, de lo que representa.

En la práctica comunicativa esto conlleva el identificarse con la imagen hasta vivir una vida virtual, con el cultivo de un aspecto corporal preestablecido por la publicidad, con la difusión e intercambio de fotografías relacionadas con todo lo que atañe al propio cuerpo, con las partidas de videojuegos, con el deporte por televisión, con el sexo por internet.

Al mismo tiempo, paradójicamente, dicha reducción de la dualidad simbólica ha llevado como nunca al ser humano a dar prioridad a la satisfacción del deseo corporal. Esta identificación con ribetes psicópatas de lo virtual y el placer corporal se observa, por ejemplo, en la filmación que hacen algunos violadores de su acto, o salvando las 
diferencias, en la necesidad de enviar fotos de objetos o experiencias de la cotidianeidad, como los platos que uno come.

Esta fusión explosiva virtualidad-realidad se ha puesto de manifiesto de forma significativa con motivo del aislamiento causado por la pandemia. Evidentemente el sujeto cultural que somos, sumado a imposiciones biopolíticas, obliga a nuestros cuerpos a limitar sus movimientos y recluirse, debido a que en ese contexto y asumiendo cierto discurso, el cuerpo expuesto representa insolidaridad, y el cuerpo aislado solidaridad. Así se soporta placenteramente permanecer en casa viviendo en el imaginario que suministran los medios de comunicación, las redes sociales, las plataformas cinematográficas, etc., debido a que esos signos son ya nuestra realidad y hasta realizan nuestra corporeidad. Pero la vivencia icónica del cuerpo como signo-realidad, y determinadas posiciones biopolíticas, llevan al mismo tiempo a otro extremo: se ha desencadenado un impulso irresistible por recuperar la relación social, la fiesta o la bebida hasta el punto de protestar lógicamente contra las autoridades por lo que se considera una opresión insoportable. Estas actitudes extremas, compatibles en un mismo sujeto, se pueden explicar de manera simple aludiendo al egocentrismo que la sociedad y la economía actual han fomentado. No falta parte de razón, pero en el fondo tiene que ver con ese desequilibrio del orden simbólico del que aquí se viene tratando, que, acuciado por la circunstancia de la epidemia, dificulta una separación reflexiva del sujeto cultural con respecto a su cuerpo.

Annie Bussière (2014) ha puesto de relieve la contradicción en el contexto de la reflexión psicoanalítica freudiano-lacaniana. En ese contexto la corporalidad se considera también en relación con lo que esta investigadora llama "la crisis de lo simbólico y la nueva economía psíquica". Si el simbolismo funciona es porque la instancia parental impone las consabidas restricciones y el sujeto, aplazando el deseo, se mantiene atento a aquello que no le es inmediatamente dado, es decir, a la otredad constitutiva de todo signo. Un comportamiento basado no en el deseo sino en el goce (jouissance) supone una quiebra del orden simbólico, porque el individuo, al no salir de su cuerpo como sujeto - no subjetivar y sujetar su cuerpo- no percibe los significados, entre otros el significado de sus actos. Esta es una tendencia que psicólogos, pedagogos y sociólogos, sin llegar a considerarla patológica, observan, y que en párrafos anteriores se ha comentado en relación con las restricciones motivadas por la pandemia. Desde ese ámbito del psicoanálisis, algunos ya desde los años setenta, propugnaron una especie de rebelión contra esa instancia parental por autoritaria y por masculina ( $c f$. con lo que hemos visto que ocurre en la semiótica, que llega a postular la libertad del significante y el placer del texto), pero desde ese mismo ámbito también se argumenta que eso abundaría en esta nueva economía psíquica que se corresponde con la nueva economía especulativa financiera donde una vez perdido el patrón oro - para la economía psíquica se habla de la pérdida del patrón falo - todo vale. Así esa defensa de la libertad y del placer serviría involuntariamente a las leyes del mercado en esta última fase del capitalismo. 


\section{A MODO DE CONCLUSIÓN: ACTUALIDAD DE LA SEMIÓTICA}

Desde la Grecia antigua, se han venido dando reflexiones sobre cómo se cifran y se comunican las ideas en los diversos ámbitos del saber, así como entre unos ámbitos y otros. Este proceder explicativo, y con potencialidad crítica, es el que Locke denominó semiótica, ya con una conciencia clara de la disciplina. Aproximadamente dos siglos después, Ch. S. Peirce y F. de Saussure forjan su forma y reconocimiento contemporáneo hasta el punto de que en los años setenta del siglo pasado la Semiótica se convierte en una moda cultural y no solo académica. Dado que en el presente trabajo se ha apuntado a la formación históricamente cambiante de la idea de actualidad y a la posibilidad de que la Semiótica no solo la recoja críticamente, sino que la integre en su corpus conceptual, se ha de concluir con una nueva pregunta: ¿Puede tener la Semiótica una conciencia de su propia actualidad? La respuesta es que no solo la viene teniendo, sino que todo lo que hasta ahora se ha escrito dentro de su campo de partida tiene actualidad si se actualiza en el contexto de la pujanza de los discursos actuales. Es decir, si esos textos disciplinares son legibles a la altura de la tercera década del siglo XXI, con los discursos actuales y a la vez haciendo legibles a estos. Tendrá que maniobrar con ellos si quiere seguir apostando por su carácter explicativo y su vocación desveladora de los mecanismos de subjetivación y socialización del ser humano en el ahora y el aquí. Todo lo que no responda a eso supone dejación, aunque el nombre de la disciplina alcance los mejores lugares en el expositor de las mercancías académicas. Ni siquiera hay que pedirle que sea crítica, porque al ver el mundo sub especie semiótica, en el peor de los casos puede servir a actitudes cínicas pero nunca supondrá llevar una venda en los ojos.

Se ha propuesto en el trabajo que aquí concluye la posibilidad de desvelar la actualidad de los discursos en diferentes escalas. En un plano amplio se ha podido comprobar que es plausible teorizar sobre un régimen del signo y de la representación, el de la arbitrariedad del signo y de la autonomía del significado respecto al referente, vigente en buena parte del siglo XX. Formulada esta lógica también por la Semiótica, como discurso que se inscribe en el mismo horizonte, su propia crítica apuntó contradictoriamente hacia una negación de dicha dualidad del signo. Buscaba una asunción más real del significante, desafiando el autoritarismo del significado convenido.

Los discursos que se vienen dando desde los años setenta, y esta puede ser la tendencia actual tras el éxito de la informática, tienden a ocupar ese polo de lo virtual, ya apuntado, donde los signos flotan con todos los significados posibles que es como no tener ninguno. El protagonismo del cuerpo en esta nueva coyuntura, que asume el lugar del significante y al mismo tiempo enajena su realidad en él, es la contradicción reveladora de la actual crisis de lo simbólico.

Esta es la tesitura que ha de afrontar la Semiótica, y, puesto que es una tesitura semiótica, no puede eludirla sin perder su actualidad y su utilidad. 


\section{REFERENCIAS BIBLIOGRÁFICAS}

ABlali, D. et DuCARD, D. (2009). Vocabulaire des études sémiotiques et sémiologiques. Paris: Champion / Presses Universitaires de Franche-Comté.

Ávila Martín, M. C. y Linares Alés, F. (2006). "Léxico y discurso de la moda". Comunicar. Revista de Medios de Comunicación y Educación 27, 35-41.

BUSSIÈRE, A. (2014). "La crise du symbolique et la nouvelle économie psychique". Sociocritique. Disponible en línea: https://www.sociocritique.fr/?La-crise-dusymbolique-et-la-nouvelle-economie-psychique-Annie-Bussiere [22/12/2020].

CROS, E. (2009). La sociocrítica. Madrid: Arco / Libros. (2021). "El estatuto de la representación y las rupturas históricas", F. Linares (trad.). CECIL 7, 9-20. Disponible en línea: https://cecil-univ.eu/c7_1/[12/12/2020].

DiJK, T. A. VAN, COMP. (2000). El discurso como estructura y como proceso. Barcelona: Gedisa.

EVERAERT-DESMEDT, N. (1990). Le processus interpretative: introduction à la sémiotique de Charles Sanders Peirce. Paris: Mardaga.

Greimas, A. J. y CourTÉs, J. (1982). Semiótica. Diccionario razonado de la teoría del lenguaje, E. Ballón Aguirre y H. Campodónico Carrión (trads.). Madrid: Gredos.

LÁzAro CARreter, F. (1981). Diccionario de términos filológicos. Madrid: Gredos.

Lotman, I. (1998). La semiosfera II. Semiótica de la cultura, del texto, de la conducta y del espacio, D. Navarro (trad.). Madrid: Cátedra.

Real Academia Española (s. f.). Banco de datos (CORDE). Corpus diacrónico del español [en línea]. Disponible en línea: http://www.rae.es [10/12/2020]

Rossi-LANDi, F. (1968). Il linguaggio come lavoro e come mercato. Milano. Bompiani.

SEgre, C. (1981). Semiótica, historia y cultura, M. Lobo y L. Badía (trads.). Barcelona: Ariel.

SElden, R. (1987). La teoría literaria contemporánea, J. G. López Guix (trad.). Barcelona: Ariel.

Wolf, M. (1987). La investigación de la comunicación de masas, C. Artal y L. Vilches (trads.). Barcelona: Paidós.

Fecha de recepción: 27/01/2021

Fecha de aceptación: 07/05/2021 